\title{
Article
}

\section{Application of Regge Theory to Astronomical Objects}

\author{
Gurgen G. Adamian ${ }^{1, *}$, Nikolai V. Antonenko ${ }^{1}{ }^{1}$, Horst Lenske ${ }^{2}\left(\mathbb{D}\right.$ and Vazgen V. Sargsyan ${ }^{1}$ \\ 1 Joint Institute for Nuclear Research, 141980 Dubna, Russia; antonenk@theor.jinr.ru (N.V.A.); \\ sargsyan@theor.jinr.ru (V.V.S.) \\ 2 Institut für Theoretische Physik, der Justus-Liebig-Universität, D-35392 Giessen, Germany; \\ horst.lenske@physik.uni-giessen.de \\ * Correspondence: adamian@theor.jinr.ru
}

check for

updates

Citation: Adamian, G.G.;

Antonenko, N.V.; Lenske, H.;

Sargsyan, V.V. Application of Regge

Theory to Astronomical Objects.

Physics 2021, 3, 669-677. https://

doi.org/10.3390/physics3030040

Received: 21 May 2021

Accepted: 31 July 2021

Published: 15 August 2021

Publisher's Note: MDPI stays neutral with regard to jurisdictional claims in published maps and institutional affiliations.

\begin{abstract}
Using the model based on the Regge-like laws, new analytical formulas are obtained for the moment of inertia, the rotation frequency, and the radius of astronomical non-exotic objects (planets, stars, galaxies, and clusters of galaxies). The rotation frequency and moment of inertia of a neutron star and the observable Universe are estimated. The estimates of the average numbers of stars and galaxies in the observable Universe are given. The Darwin instability effect in the binary systems (di-planets, di-stars, and di-galaxies) is also analyzed.
\end{abstract}

Keywords: Regge trajectories; astronomical objects; moment of inertia; Darwin instability effect

\section{Introduction}

The Regge theory [1-3] proved to be very influential in the development of elementary particle physics [4]. It is based on the analytical continuation of quantum mechanical scattering amplitude into the complex angular space [2]. The scattering amplitude is presented as a power function. As known, one of the most important properties of elementary particles is their ability to have an innate proper spin. The observed correlation between the spin and mass of hadrons shows that the heavier the hadron, the greater the spin it can have. It was revealed that the strongly interacting particles have a simple dependence of the mass on the angular momentum: the particles fall into families where the Regge trajectory functions are straight lines. The relation between the maximum spin, $S$, and mass, $M$, for all known hadrons and hadron resonances is given by a rectilinear Regge trajectory in the doubly logarithmic plane $\log _{10}(M)$ vs. $\log _{10}(S)$ (the Chew-Frautschi plot), which for large spin values can be represented as [3]:

$$
S=\hbar\left(\frac{M}{m_{p}}\right)^{2}
$$

where $\hbar$ and $m_{p}$ are the reduced Planck constant and the mass of proton, respectively.

In References [5-9], the relations, found with the Regge approach for quantum objects, have been applied to the macroscopic composite systems. As shown in References [5-9], in the general case of an $n$-dimensional astrophysical object, the relation between spin $S$ and mass $M$ of the object is the following:

$$
S=\hbar\left(\frac{M}{m_{p}}\right)^{1+1 / n} .
$$

The quasi-classical expression (2) has been derived from simple dimensionality considerations and the requirement of similarity with Equation (1) [5-9]. As seen, for the one-dimensional case, $n=1$, Equation (2) turns into Equation (1). In contrast to earlier semi-phenomenological approaches, the expressions (1) and (2) contain only fundamental constants as the parameters and are independent of any fitted empirical quantities. 
The application of Regge ideas to astrophysics has shown that the spins $S$ of planets and stars are well described by the Regge-like law for a sphere, $S \sim M^{4 / 3}, n=3$, while the spins of galaxies and clusters of galaxies obey the Regge-like law for a disk, $S \sim M^{3 / 2}$, $n=2$ [5-9]. The proposed simple Regge-like law allows us to obtain reasonable numerical values for spins of cosmic objects in a self-consistent manner, starting from planets and ending with the astronomical Universe as a whole, in an extremely wide range of masses (30 orders of magnitude) and spins (50 orders of magnitude) [5-9]. Using Equation (2), one can also obtain important formulas for the exotic neutron star and the observable Universe, which are related, respectively, to the two important cosmological Eddington and Chandrasekhar points on the cosmic analog of the Chew-Frautschi plot [8-10]. In addition, References [5-9] offer an explanation of the origin of cosmic objects and their rotation in the framework of the concept of Regge trajectories and the Ambartsumian cosmogony [11,12]. In References [13-15], an analogy has been observed between the behavior of hadrons in strong interactions at large distances and gravity. This property arises due to the possibility of exchanging a colorless combination of two-gluon with spin 2 , simulating a graviton [15]. One can also indicate a less phenomenological lines of such type researches [16-20].

The aim of the present paper is to obtain the analytical expressions for the moment of inertia, the rotation frequency, and the radius of celestial objects and to study the Darwin instability effect in a binary star or binary galaxy [21] by using the model of References [5-9], based on the concept of Regge trajectories.

\section{Planets, Stars, Galaxies, and Clusters of Galaxies}

Let us consider the individual astronomical objects such as planets, stars, galaxies, and clusters of galaxies. The general virial theorem reads:

$$
U=-2\left(E_{\mathrm{k}}+V_{\mathrm{r}}\right)
$$

where $U=-\omega G M^{2} / R$ is the gravitational potential with the Newtonian constant of gravitation, $G$, the radius, $R$, and the dimensionless structural factor, $\omega$ of the object. The value of $E_{\mathrm{k}}$ includes the kinetic energy of both the thermal motion of particles and the macroscopic motions of matter (caused by pulsations, convective currents, etc.), with the exception of the rotation energy, $V_{\mathrm{r}}=S^{2} /(2 \Im)$ of the object. The dimensionless structural factor,

$$
\omega=\int_{0}^{1} d q_{x} \frac{q_{x}}{x}
$$

is determined by the density profile, $\rho(r)$, of the object. Here, $x=r / R$, and

$$
q_{x}=M_{x} / M=\int_{0}^{r} d r^{\prime} r^{\prime 2} \rho\left(r^{\prime}\right) / \int_{0}^{R} d r^{\prime} r^{\prime 2} \rho\left(r^{\prime}\right),
$$

are the fraction of the radius and the mass fraction, respectively, of the object at a distance $r$ from the center of the object. Note that as soon as $q_{x} \leq 1$, one has $\omega \geq 1 / 2$. For the homogeneous density distribution, $\omega=3 / 5$. If the concentration of matter indefinitely increases to the center of the object, then $\omega=99 / 125 \approx 0.8$. The structure of main sequence stars is rather well distributed by polytropes of indexes $n_{0}$ from 1.5 to about 3.5 and corresponding structural factors, $\omega=3 /\left(5-n_{0}\right)$, from $6 / 7$ to about 2 .

Using Equations (2) and (3), and the observed scaling law,

$$
R=\alpha M^{m}
$$


between the radius, $R$, and the mass, $M$, of object ( $\alpha$ and $m$ are constants), one obtains the expression for the moment of inertia:

$$
\Im=-\frac{S^{2}}{U+2 E_{\mathrm{k}}}=\frac{\hbar^{2} \alpha}{\omega G M^{2-m}-2 \alpha E_{\mathrm{k}}}\left(\frac{M}{m_{p}}\right)^{2+2 / n} .
$$

On the other side,

$$
\Im=\frac{S}{\Omega}=\frac{\hbar}{\Omega}\left(\frac{M}{m_{p}}\right)^{1+1 / n}
$$

Assuming that the rotational frequency, $\Omega$, is a function of mass $M(\gamma$ and $d$ are constants),

$$
\Omega=\gamma M^{d},
$$

and using Equations (6) and (7), one can derive the following relation:

$$
m=1-\frac{1}{n}-d,
$$

connecting the constants $m$ and $n$, or the radius-mass relation

$$
R=\alpha M^{1-1 / n-d},
$$

and the new experssions:

$$
\begin{gathered}
E_{\mathrm{k}}=\beta\left(\frac{M}{m_{p}}\right)^{1+1 / n+d}, \\
\Im=\frac{\hbar^{2} \alpha}{\omega G m_{p}^{1+1 / n+d}-2 \alpha \beta}\left(\frac{M}{m_{p}}\right)^{1+1 / n-d}, \\
\gamma=\frac{\omega G m_{p}^{1+1 / n+d}}{\hbar \alpha}-\frac{2 \beta}{\hbar} .
\end{gathered}
$$

As one can see from Equation (13), the value of $\gamma$ has the dimension of frequency and, correspondingly, $d=0$ in Equation (8). Then, Equations (8)-(13) read:

$$
\begin{gathered}
m=1-\frac{1}{n}, \\
R=\alpha M^{1-1 / n}, \\
E_{\mathrm{k}}=\beta\left(\frac{M}{m_{p}}\right)^{1+1 / n}, \\
\Im=\frac{\hbar^{2} \alpha}{\omega G m_{p}^{1+1 / n}-2 \alpha \beta}\left(\frac{M}{m_{p}}\right)^{1+1 / n},
\end{gathered}
$$


and

$$
\Omega=\frac{\omega G m_{p}^{1+1 / n}}{\hbar \alpha}-\frac{2 \beta}{\hbar} .
$$

The value of $\beta$ in Equation (16) is the constant that can be extracted from the observed data of $\Omega, \alpha$, and $\omega$. As it is seen, the moment of inertia (17) depends on the dimension $n$ of the object, the classical and quantum fundamental constants $G, \hbar, m_{p}$, the dimensionless structural factor $\omega$. The constants $\alpha$ and $\beta$ are determined from the observed data. The Regge-like spin-mass Equation (2), the moment-of-inertia-mass Formula (17) (or (7)), and the radius-mass Relation (15) contain the same quantity $n$, which means that they are related to each other. One should stress that the constants $\alpha, \beta$, and $\omega$ for planets, stars, galaxies, and clusters of galaxies differ one from another.

Equations (2), (17), and (18) can be generalized by making the substitution $\hbar \rightarrow D \hbar$, where $D$ is a constant. However, as it is shown in References [5-9], spins of planets, stars, galaxies, clusters of galaxies, neutron stars and the observable Universe (see Section 3 below) are well described with $D=1$.

For star-like and planet-like objects with $n=3$, Equation (9) leads to $m=2 / 3$ and, then,

$$
R=\alpha M^{2 / 3},
$$

which is in perfect agreement with the observational data for the main sequence stars [22-28]. For main sequence stars, $\alpha=R_{\odot} / M_{\odot}^{2 / 3}$, where $R_{\odot}$ and $M_{\odot}$ are the radius and the mass of the Sun [29-31]. From Equation (12), one gets:

$$
\Im \sim M^{4 / 3} .
$$

For galaxy-like or a cluster of galaxy-like object with $n=2: m=1 / 2$ (see Equation (9)), and:

$$
R=\alpha M^{1 / 2}
$$

from Equation (10), and

$$
\Im \sim M^{3 / 2}
$$

from Equation (12). Note that the derived $m=1 / 2$ is within the observational range of $m$ of $2 / 5$ to $2 / 3$ [32]. For the galaxy-like objects, the definition of the constant $\alpha$ from the observational data is given in References [33,34].

\section{Neutron Star and Observable Universe}

The Kerr maximal spin of the rotating black hole reads:

$$
S=\frac{G M^{2}}{c}=\hbar\left(\frac{M}{m_{\mathrm{Pl}}}\right)^{2}=\hbar\left(\frac{M}{m_{p}}\right)^{2} I^{-1},
$$

where $c$ is the speed of light in vacuum, $I=\hbar c /\left(G m_{p}^{2}\right)=1.69 \times 10^{38}$ is the dimensionless combination of fundamental constants, and $m_{\mathrm{Pl}}=(\hbar c / G)^{1 / 2}=m_{p} I^{1 / 2}=1.3 \times 10^{19} m_{p}=$ $2.18 \times 10^{-8} \mathrm{~kg}$ is the Planck mass [9]. Equating Equations (2) and (23), one can derive the Chandrasekhar mass,

$$
M_{C}=m_{p} I^{3 / 2}
$$

for $n=3$, and the Eddington mass,

$$
M_{\mathrm{E}}=m_{p} I^{2}
$$


for $n=2$. The resulting masses $M_{\mathrm{C}}=2.20 \times 10^{57} m_{p}=3.46 \times 10^{30} \mathrm{~kg}$ and $M_{\mathrm{E}}=2.87 \times$ $10^{76} m_{p}=4.80 \times 10^{49} \mathrm{~kg}$ correspond to the Chandrasekhar limiting mass of a degenerate neutron star and the Eddington limiting mass of the observable Universe, respectively $[8,9]$. As seen, the neutron mass $M_{C}$ is close to the mass of the Sun, $M_{\odot}=1.99 \times 10^{30} \mathrm{~kg}$.

Taking for the average masses of stars and galaxies in the Universe, respectively, the masses of the Sun and our Galaxy $\left(M_{\mathrm{G}}=3.38 \times 10^{41} \mathrm{~kg}\right)$, and using Equation (25), one can roughly estimate the average number of galaxies,

$$
N_{\text {galaxy }} \approx \frac{M_{\mathrm{E}}}{M_{\mathrm{G}}} \approx 10^{8},
$$

and average number of stars,

$$
N_{\text {star }} \approx \frac{M_{\mathrm{E}}}{M_{\odot}} \approx 10^{19}
$$

in the observable Universe.

Substituting Equations (24) and (25) into Equation (2) leads to the limiting spins:

$$
S_{\mathrm{C}}=\hbar I^{2}
$$

for the neutron star, and

$$
S_{\mathrm{E}}=\hbar I^{3}
$$

for the observable Universe. Equation (27) predicts the rotation of the entire astronomical Universe as a whole with the spin $S_{\mathrm{E}}=2.87 \times 10^{76} \hbar=5.12 \times 10^{80} \mathrm{~J} \cdot \mathrm{s}[8,9]$. For the comparison, $S_{C}=2.20 \times 10^{57} \hbar=3.03 \times 10^{42} \mathrm{~J} \cdot \mathrm{s}$. Employing the theoretical radius-mass relation,

$$
R=G M / c^{2},
$$

of the rotating black hole, and Equations (24) and (25), one derives [10] the radius of neutron star:

$$
R_{\mathrm{C}}=r_{p} I^{1 / 2},
$$

and the radius of the observable Universe:

$$
R_{\mathrm{E}}=r_{p} I,
$$

where the factor $r_{p}=\hbar /\left(m_{p} c\right)=2.10 \times 10^{-16} \mathrm{~m}$ is the proton radius. The calculated radii (29) and (30) are: $R_{\mathrm{C}}=1.30 \times 10^{19} r_{p}=2.74 \times 10^{3} \mathrm{~m}$, and $R_{\mathrm{E}}=1.69 \times 10^{38} r_{p}=3.56 \times 10^{22}$ $\mathrm{m}$. For comparison, the radius of the Sun is $R_{\odot}=6.99 \times 10^{8} \mathrm{~m}$. The equality [9]

$$
\frac{S_{\mathrm{E}}}{R_{\mathrm{E}}^{3}}=\frac{\hbar}{r_{p}^{3}}
$$

follows from Equations (27) and (30), and implies that the spin densities of the proton and the Universe are the same within a factor of two.

Employing Equations (29) and (30), one obtains the following rotational frequencies for a neutron star and the observable Universe $\left(\omega_{p}=c / r_{p}=1.43 \times 10^{24} \mathrm{~s}^{-1}\right)$ :

$$
\begin{aligned}
& \Omega_{\mathrm{C}}=\frac{c}{R_{\mathrm{C}}}=\frac{c}{r_{p}} I^{-1 / 2}=7.68 \times 10^{-20} \frac{c}{r_{p}}=1.10 \times 10^{5} \mathrm{~s}^{-1}, \\
& \Omega_{\mathrm{E}}=\frac{c}{R_{\mathrm{E}}}=\frac{c}{r_{p}} I^{-1}=5.90 \times 10^{-39} \frac{c}{r_{p}}=8.43 \times 10^{-15} \mathrm{~s}^{-1} .
\end{aligned}
$$


Note that

$$
\frac{\Omega_{\mathrm{C}}}{\Omega_{\mathrm{E}}}=\frac{R_{\mathrm{E}}}{R_{\mathrm{C}}}=\frac{M_{\mathrm{E}}}{M_{\mathrm{C}}}=\left(\frac{S_{\mathrm{E}}}{S_{\mathrm{C}}}\right)^{1 / 2}=I^{1 / 2}=1.3 \times 10^{19} .
$$

The corresponding moments of inertia, $\Im_{\mathrm{C}}=2.76 \times 10^{37} \mathrm{~J} \cdot \mathrm{s}^{2}$ and $\Im_{\mathrm{E}}=6.08 \times 10^{94}$ $\mathrm{J} \cdot \mathrm{s}^{2}$, are calculated as follows

$$
\begin{aligned}
& \Im_{\mathrm{C}}=\frac{S_{\mathrm{C}}}{\Omega_{\mathrm{C}}}=\frac{\hbar r_{p}}{c} I^{5 / 2}, \\
& \Im_{\mathrm{E}}=\frac{S_{\mathrm{E}}}{\Omega_{\mathrm{E}}}=\frac{\hbar r_{p}}{c} I^{4},
\end{aligned}
$$

where $\hbar r_{p} / c=7.39 \times 10^{-59} \mathrm{~J} \cdot \mathrm{s}^{2}$. Note that

$$
\frac{\Im_{\mathrm{E}}}{\Im_{\mathrm{C}}}=\left(\frac{\Omega_{\mathrm{C}}}{\Omega_{\mathrm{E}}}\right)^{3}=\left(\frac{R_{\mathrm{E}}}{R_{\mathrm{C}}}\right)^{3}=\left(\frac{M_{\mathrm{E}}}{M_{\mathrm{C}}}\right)^{3}=\left(\frac{S_{\mathrm{E}}}{S_{\mathrm{C}}}\right)^{3 / 2}=I^{3 / 2}=2.2 \times 10^{57} .
$$

From Equations (3), (23), and (28), one obtains:

$$
\Im_{\mathrm{C}, \mathrm{E}}=\frac{S_{\mathrm{C}, \mathrm{E}}^{2} R_{\mathrm{C}, \mathrm{E}}}{\omega_{\mathrm{C}, \mathrm{E}} G M_{\mathrm{C}, \mathrm{E}}^{2}}=\frac{M_{\mathrm{C}, \mathrm{E}} R_{\mathrm{C}, \mathrm{E}}^{2}}{\omega_{\mathrm{C}, \mathrm{E}}} .
$$

From comparing these equations with Equations (34) and (35), it follows that the dimensionless structural factors for the neutron star and the observable Universe are equal to the unity, $\omega_{\mathrm{C}}=\omega_{\mathrm{E}}=1$, and $E_{\mathrm{k}}=\beta=0$. Surprisingly, the moments of inertia (34) and (35) are larger than corresponding rigid-body moments of inertia.

Counting the average age of the Universe on the order of 14 billion years and using Equation (33), one derives a numerical value of the angular velocity of rotation of the Universe:

$$
\Omega_{\mathrm{E}}=4 \times 10^{3} \frac{1}{\text { age }- \text { of }- \text { Universe }} .
$$

In other words, the total rotation time of the observable Universe is approximately $10^{7}$ years, which is about $10^{3}$ times less than the average age of the Universe. In Reference [9], the estimated frequency estimate is about six orders of magnitude less than the value given by Equation (36) due to the difference in the moments of inertia used in the calculations.

\section{Darwin Instability Effect in Binary Systems}

Now let us apply the Regge-theory to the astronomical compact binary systems. When the mass ratio in the compact binary star is extreme enough for the Darwin instability [21], a merger of the binary components starts that triggers the outburst in a red nova [35]. The Darwin instability happens when the spin of the system is more than one-third of the orbital angular momentum. This instability plays a role once the mass ratio becomes small enough that the companion star can no longer keep the primary star synchronously rotating via the tidal interaction. The angular momentum transferred from the binary orbit to the intrinsic spin changes the orbit and leads to a runaway. For most of the massive primary stars, this occurs at the mass ratio $q=M_{2} / M_{1}<0.1$ [36].

The total angular momentum, $\mathbf{J}$, of the binary system is the sum of the orbital angular momentum, $\mathbf{L}$, and the spins, $\mathbf{S}_{k}, k=1,2$, of the individual components:

$$
\mathbf{J}=\mathbf{L}+\mathbf{S}_{1}+\mathbf{S}_{2}
$$


The $J$ and $S_{k}$ are expressed using the Regge-like laws $[5,8,9]$ :

$$
J=\hbar\left(\frac{M}{m_{p}}\right)^{1+1 / n},
$$

and

$$
S_{k}=\hbar\left(\frac{M_{k}}{m_{p}}\right)^{1+1 / n},
$$

where $M_{k}, k=1,2$, and $M=M_{1}+M_{2}$ are masses of the binary components and the total mass of the system, respectively. Then, the maximum (the antiparallel orbital and spin angular momenta) and minimum (the parallel orbital and spin angular momenta) orbital angular momenta are:

$$
L_{\max }=J+S_{1}+S_{2},
$$

and

$$
L_{\min }=J-S_{1}-S_{2},
$$

respectively. Using Equations (37)-(41), one derives:

$$
\frac{S_{1}+S_{2}}{L_{\min }}=\frac{1+q^{1+1 / n}}{(1+q)^{1+1 / n}-q^{1+1 / n}-1},
$$

and

$$
\frac{S_{1}+S_{2}}{L_{\max }}=\frac{1+q^{1+1 / n}}{(1+q)^{1+1 / n}+q^{1+1 / n}+1} .
$$

For $q=1$ (symmetric binary system) and $n>1$, one has:

$$
\frac{S_{1}+S_{2}}{L_{\min }}=\frac{1}{2^{1 / n}-1}>1,
$$

and

$$
\frac{S_{1}+S_{2}}{L_{\max }}=\frac{1}{2^{1 / n}+1}>\frac{1}{3} .
$$

For a symmetric binary star or binary planet $(n=3)$, or binary galaxy $(n=2)$ with $q=1,\left(S_{1}+S_{2}\right) / L_{\max } \approx 0.44$ and 0.41 , respectively. At $q \rightarrow 0$ :

$$
\frac{S_{1}+S_{2}}{L_{\min }} \rightarrow \infty,
$$

and

$$
\frac{S_{1}+S_{2}}{L_{\max }} \rightarrow \frac{1}{2} .
$$

As follows from these two expressions, for a very asymmetric binary system, the ratios $\left(S_{1}+S_{2}\right) / L_{\max , \min }$ are almost independent of the value of $n$. According to Reference [36], the Darwin instability can occur when the binary mass ratio is very small $(q<0.1)$ or the mass asymmetry is very large. The ratios $\left(S_{1}+S_{2}\right) / L_{\max }$ and $\left(S_{1}+S_{2}\right) / L_{\min }$, continuously increase with decreasing $q$ from 1 to 0 . As soon as the absolute values of the ratios are larger than $1 / 3$, all possible binary stars or binary planets, or binary galaxies, independently of their mass ratios $q$, have to have the Darwin instability $\left(S_{1}+S_{2} \geq L / 3\right)$ [21] and, hence, to merge. However, the observations do not support this conclusion which probably means that there is no Darwin instability effect in such binary systems and, then, the mechanism of merging has other origins to be searched for. 
As follows, in the cases of antiparallel spins with $L_{12}=J+S_{1}-S_{2}$ and $L_{21}=$ $J-S_{1}+S_{2}$, the ratios $\left|S_{2}-S_{1}\right| / L_{12}$ and $\left|S_{1}-S_{2}\right| / L_{21}$ are larger than $1 / 3$ for the asymmetric binaries with $q \leq 1 / 3$ [37].

\section{Summary}

Within the model [5-9], based on a concept of Regge trajectories, new analytical expressions for the moment of inertia, the rotation frequency, and the radius of astronomical objects (stars, planets, galaxies, and clusters of galaxies) are derived. The moment of inertia is found to depend on the total mass, the dimension of the object, the dimensionless structural factor, the classical, the quantum fundamental constants such as the Newtonian constant of gravitation, the reduced Planck constant and the mass of proton, and the two constants to be extracted from the observed data.

The expressions for the rotation frequencies, and moments of inertia, of neutron star and the observed Universe are derived. The estimate of the speed of rotation of the Universe is found to be of about (5-7) orders of magnitude larger than that of estimates. The rotation time of the observable Universe is found to be about $10^{7}$ years. The estimated average numbers of galaxies and stars in the observable Universe are obtained to be $\sim 10^{8}$ and $\sim 10^{19}$, respectively.

Employing the Regge-like laws, it is also shown that all possible binary stars (binary planets) or binary galaxies satisfy the Darwin instability condition, which contradicts the observations. This conclusion is not sensitive to the parameters of the model. Therefore, other possible mechanisms that trigger the merger of the contact binary components to be searched for.

Author Contributions: Conceptualization, G.G.A., N.V.A., H.L. and V.V.S.; data curation, G.G.A., N.V.A., H.L. and V.V.S.; formal analysis, G.G.A., N.V.A., H.L. and V.V.S.; funding acquisition, G.G.A., N.V.A., H.L. and V.V.S.; methodology, G.G.A., N.V.A., H.L. and V.V.S.; software, V.V.S.; supervision, V.V.S.; visualization, V.V.S.; writing—original draft, G.G.A.; writing—review \& editing, N.V.A., H.L. and V.V.S. All authors have read and agreed to the published version of the manuscript.

Funding: This work was partially supported by Russian Foundation for Basic Research (Grant 20-02-00176, Moscow) and Deutsche Forschungsgemeinschaft (Grant Le439/16, Bonn).

Acknowledgments: V.V.S. acknowledges the Alexander von Humboldt-Stiftung (Bonn).

Conflicts of Interest: The authors declare no conflict of interest.

\section{References}

1. Regge, T. Introduction to complex orbital momenta. Nuovo Cim. 1959, 14, 951-976. [CrossRef]

2. Bottino, A.; Longoni, A.M.; Regge, T. Potential scattering for complex energy and angular momentum. Nuovo Cim. 1962, 23, 954-1004. [CrossRef]

3. Chew, G.F.; Frautschi, S.C. Principle of equivalence for all strongly interacting particles within the S-matrix framework. Phys. Rev. Lett. 1961, 7, 394-397. [CrossRef]

4. Collins, P.D.B. An Introduction to Regge Theory and High Energy Physics; Cambridge University Press: Cambridge, UK, 1977. [CrossRef]

5. Muradyan, R.M. Origin of galactic rotation in the Ambartsumyan cosmogony. Astrophys. 1975, 11, 161-168. [CrossRef]

6. Muradyan, R.M. Cosmic coincidences and rotation of the metagalaxy. Astrophys. 1977, 13, 32-34. [CrossRef]

7. Muradyan, R.M. Origin of magnetic fields and superdense cosmogony. Astrophys. 1978, 14, 240-244. [CrossRef]

8. Muradian, R.M. The primeval hadron: Origin of stars, galaxies and astronomical universe. Astrophys. Space Sci. 1980, 69, 339-351. [CrossRef]

9. Muradian, R.M. The Regge law for heavenly bodies. Phys. Part. Nucl. 1997, 28, 471-482. [CrossRef]

10. Muradian, R.M.; Carneiro, S.; Marques, R. Radius-mass scaling laws for celestial bodies. arXiv 1999, arXiv:astro-ph/9907129.

11. Institut International de Physique Solvay. On the evolution of galaxies. In La Structure et I'Evolution de I'Universe, Proceedings of the

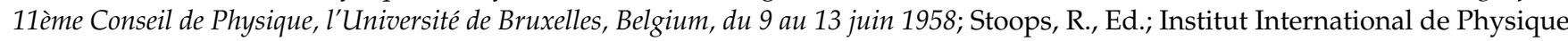
Solvay: Brussels, Belgium, 1958; pp. 241-279. Available online: http://www.solvayinstitutes.be/pdf/Proceedings_Physics/1958 .pdf (accessed on 11 August 2021)

12. Ambartsumian, V.A. Instability phenomena in systems of galaxies. Astrophys. J. 1961, 66, 536-540. [CrossRef] 
13. De Sabbata, V.; Gasperini, M. Strong gravity with torsion: Some cosmological deduction. Lett. Nuovo Cim. 1979, $25,489-492$. [CrossRef]

14. De Sabbata, V.; Sivaram, C. Strong gravity as the connecting link underlying universal relations between angular momenta of celestial bodies and spin of elementary particles. Nuovo Cim. 1988, 100, 919-926. [CrossRef]

15. Ne'eman, Y.; Sijacki, D. Proof of pseudo-gravity as QCD approximation for the hadron IR region and $J \sim M^{2}$ Regge trajectories. Phys. Lett. B 1992, 276, 173-178. [CrossRef]

16. Russo, J.G. Strong magnetic limit of string theory. J. High Energy Phys. 2005, 06, 005-013. [CrossRef]

17. Bunster, C.; Portugues, R.; Cnockaert, C.; Henneaux M. Monopoles for gravitation and for higher spin fields. Phys. Rev. D 2006, 73, 105014-105030. [CrossRef]

18. Santamato, E.; De Martini, F. Derivation of the Dirac equation by conformal differential geometry. Fond. Phys. 2013, 43, 631-641. [CrossRef]

19. Abramovsky, V.A. Abramovsky-Gribov-Kancheli Theorem in the physics of black holes. Physics 2019, 1, 253-270. [CrossRef]

20. Tokuda, J.; Aoki, K.; Hirano, S. Gravitational positivity bounds. J. High Energy Phys. 2020, 11, 054-091. [CrossRef]

21. Darwin, G.H. The determination of the secular effects of tidal friction by a graphical method. Proc. R. Soc. London 1879, 29, 168-181. Available online: https:/ / www.jstor.org/stable/113751 (accessed on 11 August 2021)

22. Vasiliev, B.V. Physics of stars and measurement data: Part I. Univ. J. Phys. Applic. 2014, 8, 257-262. [CrossRef]

23. Vasiliev, B.V. Physics of stars and measurement data: Part II. Univ. J. Phys. Applic. 2014, 8, 284-301. [CrossRef]

24. Vasiliev, B.V. Physics of stars and measurement data: Part III. Univ. J. Phys. Applic. 2014, 8, 328-343. [CrossRef]

25. Vasiliev, B.V. Star physics and measurement data. J. Mod. Phys. 2018, 9, 1906-1934. [CrossRef]

26. Cherepashchuk, A.M. Close Binary Stars; Fizmatlit: Moscow, Russia, 2012; Volume 1. (In Russian)

27. Cherepashchuk, A.M. Close Binary Stars; Fizmatlit: Moscow, Russia, 2013; Volume 2. (In Russian)

28. Eggleton, P.P. Evolutionary Processes in Binary and Multiple Stars; Cambridge University Press: Cambridge, UK, 2006. [CrossRef]

29. Sargsyan, V.V.; Lenske, H.; Adamian, G.G.; Antonenko, N.V. From dinuclear systems to close binary stars: Application to source of energy in the universe. Int. J. Mod. Phys. E 2018, 27, 1850093. [CrossRef]

30. Sargsyan, V.V.; Lenske, H.; Adamian, G.G.; Antonenko, N.V. From dinuclear systems to close binary stars: Application to mass transfer. Int. J. Mod. Phys. E 2018, 27, 1850063. [CrossRef]

31. Sargsyan, V.V.; Lenske, H.; Adamian, G.G.; Antonenko, N.V. Origin of the orbital period change in contact binary stars. Int. J. Mod. Phys. E 2019, 28, 1950044. [CrossRef]

32. Karachentsev, I.D. Binary Galaxies; Nauka: Moscow, Russia, 1987. (In Russian)

33. Sargsyan, V.V.; Lenske, H.; Adamian, G.G.; Antonenko, N.V. Close binary galaxies: Application to source of energy and expansion in Universe. Int. J. Mod. Phys. E 2019, 28, 1950031. [CrossRef]

34. Sargsyan, V.V.; Lenske, H.; Adamian, G.G.; Antonenko, N.V. From dinuclear systems to close binary stars and galaxies. Phys. Atom. Nucl. 2020, 83, 60-68. [CrossRef]

35. Tylenda, R.; Hajduk, M.; Kamiński, T.; Udalski, A; Soszyński, I.; Szymański, M.K.; Kubiak, M.; Pietrzyński, G.; Poleski, R.; Wyrzykowski, Ł.; et al. V1309 Scorpii: Merger of a contact binary. Astron. Astrophys. 2011, 528, A114. [CrossRef]

36. Rasio, F.A. The minimum mass ratio of W ursae majoris binaries. Astrophys. J. Lett. 1995, 444, L41. [CrossRef]

37. Adamian, G.G.; Antonenko, N.V.; Lenske, H.; Sargsyan, V.V. Stability of macroscopic binary systems. Commun. Theor. Phys. 2019, 71, 1335-1340. [CrossRef] 